\title{
Precio del suelo dado por la oferta del mercado y el avalúo municipal: Relaciones y distribución espacial en la ciudad de Cuenca
}

\author{
Price of land determined by the market supply and the municipal appraisal: \\ Relations and spatial distribution in the city of Cuenca
}

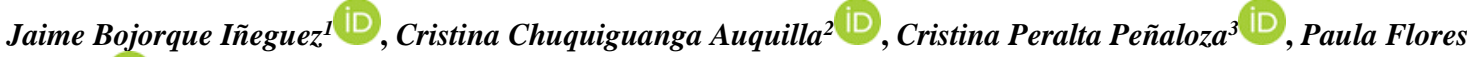 \\ Chafla $^{3}$ iD \\ ${ }^{1}$ Profesor de la Facultad de Ingeniería, Departamento de Ingeniería Civil, Universidad de Cuenca, Av. 12 de Abril y Agustín \\ Cuenca, Cuenca, Ecuador, 01.01.168. \\ ${ }^{2}$ Profesora de la Facultad de Arquitectura y Urbanismo, Universidad de Cuenca, Av. 12 de Abril y Agustín Cuenca, Cuenca, \\ Ecuador, 01.01.168. \\ ${ }^{3}$ Arquitecta, egresada de la Facultad de Arquitectura y Urbanismo, Universidad de Cuenca.
}

Autor para correspondencia: jaime.bojorque@ucuenca.edu.ec

Fecha de recepción: 3 de septiembre de 2020 - Fecha de aceptación: 19 de septiembre de 2020

\section{RESUMEN}

En el presente documento se analiza la relación entre el precio de mercado y el valor catastral de los lotes baldíos, urbanizados y de propiedad horizontal, y su distribución espacial dentro del área urbana de la ciudad de Cuenca, con una superficie aproximada de $74 \mathrm{~km}^{2}$. El valor catastral de los lotes se obtuvo del portal del Gobierno Municipal Autónomo Descentralizado del Cantón Cuenca. Mientras que el precio de mercado se consiguió de las ofertas inmobiliarias publicadas en el período septiembre 2019 a marzo de 2020. De los 100,203 lotes, dentro del área de estudio, 994 lotes, de los cuales se encuentran disponibles el precio de mercado y el valor catastral, fueron incluidos en el estudio. En total, se utilizaron en el análisis 421 lotes sin construir, 518 parcelas construidas y 55 propiedades horizontales. El precio del suelo según la tasación municipal equivale, en promedio, al $54.15 \%$ del precio de mercado, lo que demuestra que el valor catastral de las propiedades está subvalorado. La autocorrelación espacial entre el precio de mercado y el valor catastral se derivó utilizando las estadísticas I de Moran y Gi*. Se determinaron lotes con precio de mercado alto con respecto al valor catastral, el 5\% de los predios mostraron valores superiores al 4.82 con un máximo de 11.39. Considerando estos aspectos, los municipios, dado que la sociedad cambia a un ritmo acelerado, deben modernizar y renovar el sistema catastral, que tradicionalmente se considera que funciona bien, pero en muchos casos está desactualizado.

Palabras clave: Precio del suelo, precio de mercado, avalúo municipal, Cuenca.

\begin{abstract}
The paper analyzes the relationship between the market price and the cadastral value of vacant lots, built-up lots, and horizontal property, and its spatial distribution within the urban area of the city of Cuenca, covering an area of approximately $74 \mathrm{~km}^{2}$. The cadastral value of the lots was obtained from the Autonomous Decentralized Municipal Government of the Canton Cuenca. Whereas, the market price was obtained from the published real estate offers in the period September 2019-March 2020. Of the 100,203 lots within the study area, 994 lots, of which the market price and cadastral value are available, were retained in the study. In total 421 unbuilt-plots, 518 built-plots, and 55 horizontal properties were used in the analysis. The land price by the municipal appraisal equals on average $54.15 \%$ of the market price, showing that the cadastral value of the properties is undervalued. The spatial autocorrelation between market price and the cadastral value was derived using Moran's I and Gi* statistics. Lots were observed with a high market price, $5 \%$ of the properties presented values higher than 4.82 with a maximum of 11.39. These high values do not have a clear explanation, except speculation given by the market. Given the difference between both measures, municipalities, given society changes at a rapid pace, should modernize and renew the cadastral system, which traditionally is seen as well-functioning but in many cases is outdated.
\end{abstract}

Keywords: Land price, market price, municipal appraisal, Cuenca. 


\section{INTRODUCCIÓN}

El suelo es fundamental para el progreso de los ciudadanos, no solo porque la vivienda, la propiedad comercial, el espacio público y el transporte se desarrollan sobre este, sino además afecta al mercado laboral y de productos. A pesar de este rol importante del suelo, es considerado propiedad privada en casi todo el mundo, alcanzando precios muy altos. Pero ¿Cuál es el valor referencial del suelo urbano?, esta es una pregunta muy difícil de contestar y alrededor de la cual se han desarrollado varias teorías, por ejemplo, la Teoría de la Renta del Suelo Urbano (Jaramillo, 2009).

El suelo es un bien único y no reproducible; escaso por naturaleza y un monopolio sobre el cual se generan valores diferenciales a lo largo de la estructura urbana. En este sentido, adquiere sus cualidades en función de la inversión económica que realiza la población para hacerlo habitable y de atributos externos a él que califican su contexto (Granda, Cuesta, \& Villagomez, 2017). Los bienes inmuebles representan un bien agrupado que consiste en suelo y capital incorporado. Con respecto al primero, las influencias de las fuerzas económicas, sociales y legales se reflejan en los valores del suelo. Por lo tanto, estos guían la formación de las ciudades y generan ingresos para los gobiernos locales (Womack, 2015). Los precios son, por lo general, una expresión del valor que el mercado otorga a los bienes según sus características. Las particularidades que más influyen en el precio del suelo urbano son las extrínsecas, como la ubicación relativa que tiene en la distribución urbana con respecto a otros lotes, más que sus características intrínsecas, como el tamaño o la forma del terreno (Erba, 2013). La naturaleza de la propiedad del suelo, el método de realizar las transacciones y la falta de información pública suman a las imperfecciones del mercado inmobiliario. La mayoría de las negociaciones se realizan de forma privada, e incluso cuando se conocen los resultados de las transacciones, no son útiles debido a la ausencia de información detallada sobre cuestiones como el tamaño y el estado de los edificios y el título legal.

Se debe tener en cuenta, sin embargo, que el método de valoración a elegir depende de los fines o del interés por obtener dicha información. Así, los propietarios estarán atraídos por conocer el precio de venta o de alquiler que pueden solicitar a su propiedad; los agentes inmobiliarios, generalmente, analizan los precios de oferta según la ubicación que genere mayor rentabilidad; los compradores (o inquilinos) indagan el mercado para saber cuánto deben pagar; los acreedores hipotecarios se encuentran interesados por el valor de la propiedad para la garantía del préstamo y los dueños por conocer sobre su derecho a compensación e impuestos. De aquí que, el enfoque para la estimación del valor en un caso puede ser diferente a otro y, con el tiempo, se han desarrollado enfoques o métodos de valoración separados (Shapiro, Mackmin, \& Sams, 2013). El precio de un bien se puede calcular en función de sus diferentes características, sean estas cualidades o cantidades. En este concepto se basa el modelo hedónico de valuación y también el de costo de reposición. El modelo hedónico es adecuado al incluir un variado número de factores en la estimación del precio (Paterson \& Boyle, 2002), sin embargo, tiene limitaciones en el sentido de que no considera los efectos espaciales que ocurren en cada región, las cuales se superan con econometría espacial (Kim \& Kim, 2016), y la necesidad de contar con una amplia información de las características específicas de cada predio. Adicionalmente, el valor de un inmueble que se encuentre bajo explotación económica puede ser calculado en función de las expectativas de rentas. En este sentido, la oferta del mercado está ligada a la demanda que genera la correcta explotación de las potencialidades del terreno (Erba, 2013).

En el Ecuador, el Código Orgánico de Organización Territorial, Autonomía y Descentralización (COOTAD) orienta la definición de políticas y mecanismos para el desarrollo territorial. En su Art. 495, menciona que, el costo de la propiedad se establecerá mediante la suma del valor del suelo y de las construcciones que se hayan edificado. El término "valor del suelo" hace referencia al precio unitario de suelo, determinado por un proceso de comparación con precios de venta de inmuebles de condiciones similares u homogéneas del mismo sector, multiplicado por la superficie del predio. Este valor sirve de base para la determinación de impuestos y para otros efectos tributarios y no tributarios a partir del cual las municipalidades pueden establecer criterios de valor de los inmuebles dados por intervención pública, social u otro factor de incremento (COOTAD, 2010). Es importante destacar que, este documento normativo, plantea una estimación simplificada para el cálculo del costo del terreno, dejando a la especulación tomar espacio, sin realizar un verdadero análisis técnico del precio que puede tener un suelo considerando un adecuado desarrollo territorial.

En condiciones normales, los precios de mercado son el resultado de estimaciones realizadas por compradores y vendedores sobre la base de precios previamente pagados por otras propiedades similares. Sin embargo, en momentos en que las condiciones del mercado no son estables, pueden surgir diferencias más significativas. Por ello, es importante, ir más allá de la oferta y la demanda para realizar una estimación del valor del suelo, debido a los múltiples aspectos de desarrollo que están involucrados en este análisis, por ejemplo, el derecho a vivienda digna. En el estudio realizado en la ciudad de Tena-Ecuador, Granda, Cuesta, \& Villagomez (2017) muestran que la valoración de la tierra urbana es muy subjetiva, pues los criterios que caracterizan a cada uno de los predios urbanos son, en su mayoría, provenientes de una cualificación urbanística. Esto se evidencia de igual manera en otras regiones del país. Así, el precio, como expresión de la relación del interés que tiene un determinado sujeto con respecto al predio urbano, viene a representar el soporte para la decisión individual de las partes intervinientes en el proceso de valoración; es decir, constituye normalmente las fronteras de negociación.

En este complicado proceso de valoración del suelo, es importante resaltar las estimaciones que realizan los Gobiernos Autónomos Descentralizados Municipales en Ecuador para el cobro del impuesto al predio urbano. Por ejemplo, en Cuenca, el $15.07 \%$ del total recaudado por concepto de tributos se relaciona con cobros de impuestos por el predio (Quille \& Quizhipi, 2011). Vale la pena resaltar que, el precio comercial del suelo difiere en gran medida del avalúo municipal, generalmente la valuación masiva presenta problemas de subvaluación con respecto al valor real comercial de los bienes inmuebles ya sea por razones técnicas o en su mayoría por cuestiones políticas (Borrero, 2010), inclusivo en América Latina se ha identificado que los valores catastrales suelen ser 
manipulados para privilegiar a ciertos grupos (Erba, 2007).

Las municipales, en general, enfrentan dificultades para determinar el valor catastral, por un lado, mantener un catastro vigente con información cartográfica y temática demanda altos costos, más aún cuando la actualización no es parte de la actividad diaria, y por otro, contar con información real de las transacciones inmobiliarias requiere trabajar en nuevas metodologías que agiliten el proceso, de aquí que la aplicación de modelos predictivos para la valuación masiva, y a la vez, la creación de observatorios en las que se recopile información de los precios de venta, puede ser una alternativa (LozanoGracia \& Anselin, 2012).

Se debe de considerar, adicionalmente, que el precio del suelo urbano es una variable espacial auto correlacionada tanto a nivel de ciudad como de región, es decir, depende de su entorno local y regional. Muchas investigaciones han verificado la autocorrelación del valor inmobiliario urbano a nivel de ciudad, identificándose que la autocorrelación espacial del precio del suelo urbano es causada principalmente por la autocorrelación espacial económica (Conway et al., 2010; Jiao \& Liu, 2012; Xiao et al., 2017). Pero su grado de autocorrelación espacial es menor que los factores económicos porque el precio de la tierra de referencia urbana también está influenciado por otros factores locales especiales, como: la población, la infraestructura de la ciudad, el suministro de tierras, entre otros (Jiao \& Liu, 2012). La autocorrelación espacial es una evaluación de la correlación de una variable en referencia a la ubicación espacial de la variable. Para el análisis de la autocorrelación espacial se pueden emplean estadísticas como la I de Moran y Gi* (Liu, Li, Sun, \& Ma, 2006).

A nivel mundial, el avalúo del suelo se toma como un referencial para imponer impuestos, así como una base para establecer y ejecutar diversas políticas gubernamentales. De aquí que es necesario realizar investigaciones en esta área y sustentar la legislación en la aplicación de modelos estadísticos espaciales para estimar los precios oficiales del suelo.

El objetivo de este documento es analizar la relación existente entre los precios del suelo dados por la oferta de mercado y el precio del suelo indicado en el avalúo municipal, e identificar posibles relaciones espaciales. Se parte de más de mil precios obtenidos de un intenso trabajo de campo durante el periodo septiembre 2019 a marzo 2020, y se contrastan con los avalúos municipales vigentes de la ciudad de Cuenca. Finalmente, apoyado en la metodología del I de Moran local y agrupamiento espacial $\mathrm{Gi}^{*}$, se analiza el comportamiento espacial de la relación entre precio del mercado y el valor de avalúo.

\section{MATERIALES Y MÉTODOS}

\subsection{Zona de estudio}

La zona de estudio corresponde al área urbana de Cuenca en Ecuador, con una extensión aproximada de $74 \mathrm{~km}^{2}$. El cantón Cuenca según el VII Censo de Población y VI de Vivienda del 2010, realizado por el Instituto Nacional de Estadísticas y Censos (INEC), tenía 505,585 habitantes, de los cuales 331,888 habitantes (65.6\% del cantón) residían en el área urbana, y 173,697 habitantes (34.4\%) en el área rural. La Secretaria Nacional de Planificación, estima al 2020 para la ciudad, una población de 418,152 habitantes. El total de viviendas en la ciudad de Cuenca se determinó en 89,789 (INEC, 2010), y el porcentaje de hogares que habitan en viviendas propias en el $45.53 \%$ (PDOT Cuenca, 2016). Para el año 2020 existen más de 100,000 predios registrados en el catastro municipal.

\subsection{Recopilación de información \\ Ofertas del mercado}

Los precios del suelo se derivaron de la oferta inmobiliaria en la ciudad de Cuenca entre septiembre 2019 a marzo 2020. Se emplearon diversas fuentes de información entre las que destacan: publicidad (letreros), redes sociales e inmobiliarias. La publicidad fue la mayor fuente de datos, y fundamentalmente esta información fue recogida en un recorrido por la ciudad, en el cual se realizó un registro fotográfico de los diferentes letreros mediante la aplicación Google fotos, que permite administrar información relativa a la hora y ubicación de la fotografía. Las redes sociales e inmobiliarias, a su vez, fueron la segunda fuente de información; la búsqueda se realizó principalmente en páginas relacionadas a venta de propiedades tales como: OLX, Marketplace de Facebook, Plusvalía, y páginas web de inmobiliarias. Es necesario mencionar que, las fuentes relativas a publicidad en periódicos (prensa) fue utilizada de manera esporádica, debido a la repetición continua de dichos anuncios en los medios digitales mencionados anteriormente. La información levantada según el tipo de propiedad comprende: la superficie del lote $\left(\mathrm{m}^{2}\right)$, el precio del lote, y observaciones (en caso de que la situación amerite). Para lotes con edificaciones, información adicional como la superficie de construcción $\left(\mathrm{m}^{2}\right)$, el precio de la propiedad, el material de la estructura, y año de construcción, fue levantada. Adicional a los datos indicados, se identificó la clave catastral, cuyo fin es facilitar el geoprocesamiento de los datos, la información fue obtenida del GeoportalWeb Municipal.

Para la determinar el tamaño de la muestra, la unidad de análisis es el predio urbano (se cuenta con 100,203 lotes en la base de datos) y se considera el área de estudio el límite urbano, aprobada en la ordenanza que sanciona el Plan de Desarrollo y Ordenamiento Territorial de Cuenca (GAD Cuenca, 1998). La metodología empleada es el muestreo estratificado con afijación proporcional (Scheaffer, Mendenhall, Ott, \& Gerow, 2012), teniendo como universo poblacional al suelo urbano, se establece el peso de los estratos (sectores de planeamiento), fijando la cantidad de datos a levantar en cada sector para efectos del análisis. Se excluyeron aquellos sectores considerados "especiales" que por su uso de suelo no forman parte de la muestra, como se detallan a continuación: Los sectores S24, S-14 y N-10, que corresponden a los predios de la Universidad de Cuenca, de la Universidad del Azuay y de la Universidad Politécnica Salesiana, respectivamente. Los sectores E-19, E-26 y N-7A, pertenecientes a las estaciones militares de Batallón de Infantería $\mathrm{N}^{\circ} 9$ Cayambe, Grupo de caballería mecanizada $\mathrm{N}^{\circ} 6$ General Dávalos y Tercera Zona Militar, respectivamente; y, el Sector E-24, correspondiente a las lagunas de oxidación del sistema de alcantarillado de la ciudad.

Para el cálculo de la muestra $(n)$ se considera; el tamaño de la población $(N)$, el nivel de significancia $(z)$, el error 
esperado (e), la probabilidad de éxito $(p)$ y fracaso $(q)$, según la ecuación siguiente:

$$
n=\frac{N z^{2} p q}{e^{2}(N-1)+z^{2} p q}
$$

Para cada sector de planeación se determina el número muestral del estrato $\left(n_{e}\right)$ en función de la proporción del estrato $\left(p_{e}\right)$, como:

$$
n_{e}=\frac{p_{e}}{100} * n
$$

\section{Avalúos municipales}

Los avalúos municipales de los predios se obtuvieron de la página institucional del GAD Municipalidad de Cuenca $^{1}$, en la cual se revisó el informe predial que constituye un reporte con datos que describen las principales características. Los avalúos se relacionan con el plano de valor del suelo urbano de la cabecera cantonal bienio 2020-2021 (GAD Cuenca, 2019). La información levantada corresponde al periodo febrero a marzo 2020. Para relacionar, y posteriormente contrastar la información levantada (precio de mercado) con el avalúo municipal, se emplea la clave catastral. Vale señalar que, en algunos casos, el área del terreno registrado en el municipio, así como el área de construcción no corresponden a la superficie señalada por el vendedor. Estas discrepancias se deben, entre otros aspectos, a la falta de actualización de la base de datos municipal, desconocimiento del oferente, lotizaciones aún sin divisiones registradas, por señalar las identificadas. Por ello, se considera una variación máxima de $15 \%$ del área del lote, entre la registrada y la proporcionada por el oferente, para incluir el predio en el análisis.

\subsection{Determinación del precio de suelo por metro cuadrado}

El proceso para la obtención de valores de suelo de la oferta inmobiliaria en la ciudad de Cuenca se realiza según el tipo de bien registrado, sea estos: predios sin edificación, con edificación y propiedad horizontal. Con el fin de identificar posibles variaciones en los valores debido al tipo de lote se emplean técnicas estadísticas para evaluar el comportamiento de grupos de valores. Las técnicas de análisis estadístico pueden determinar si los datos son aleatorios o se pueden agrupar en conjuntos similares. Quizás el método de análisis más útil es la prueba Student t-test, que se usa para identificar si la diferencia entre valores promedio es lo suficientemente grande como para implicar que los valores medios son diferentes (Larsen \& Marx, 2006).

\section{Predios sin edificación}

Dado que el predio no incluye construcción, el precio por metro cuadrado $\left(\mathrm{m}^{2}\right)$ del suelo es el precio divido por su área.

\section{Predios con edificación}

El proceso empleado consiste en valorar la edificación en función de sus características, el estado y las condiciones

\footnotetext{
${ }^{1}$ https://enlinea.cuenca.gob.ec/\#/informe-predial
}

particulares indicadas por el propietario o intermediario. La estimación del costo inicial de las edificaciones se realiza a partir de las especificaciones técnicoconstructivas predominantes en la propiedad, a saber; materiales de la estructura, paredes, piso y cubierta estableciendo un costo por $\mathrm{m}^{2}$ según el tipo de acabados, el cual fue determinado por la experiencia de los constructores de la zona. Posteriormente, para determinar el precio final de la construcción, se considera que, al finalizar la vida útil de una edificación, el valor residual corresponde al $30 \%$ del precio inicial. La vida útil depende del tipo de material; para estructuras de hormigón, metálica y compuesta es de 50 años, para adobe y madera 40 años, y para bareque 30 años. Las correspondientes tasas de depreciación anual, según el tipo de estructura, varía entre $2.38 \%$ y $2.97 \%$. Vale la pena señalar que los datos de precios de los materiales utilizados corresponden al periodo enero a febrero 2020.

El valor de la edificación es el resultado de multiplicar el precio base de la construcción por la tasa de depreciación anual elevado a la edad de la construcción en años, según la siguiente ecuación:

$$
P_{e}=P_{b}(1-t)^{e}
$$

donde $P_{e}=$ precio final de la construcción, $P_{b}=$ precio base de la construcción, $t=$ tasa de depreciación anual, y $e=$ edad de la construcción en años.

Así, el precio por $\mathrm{m}^{2}$ será el resultado de restar el precio solicitado por el oferente menos el costo de la construcción y dividido por el área total. En casos donde la construcción es obsoleta, es decir, ya ha alcanzado su vida útil, el precio del $\mathrm{m}^{2}$ de construcción representa únicamente el valor del terreno. Debido a la complejidad y a las particularidades de las edificaciones en venta, es necesario realizar un proceso intermedio para la valuación de la propiedad: i) Para las propiedades cuya edificación se encuentra en obra gris, el $\mathrm{m}^{2}$ de construcción representa el 50\% del valor estimado del precio base de edificaciones nuevas, y ii) Se consideró la fuente de la información, es decir, si la propiedad es ofertada por una inmobiliaria, el precio final de la construcción se resta su valor en un $4 \%$, este porcentaje representa la ganancia del corredor de bienes raíces por la venta de la edificación.

\section{Propiedad horizontal}

El cálculo para estos predios es similar al de predios con edificación. A partir del precio (precio promedio, si existen más de una oferta en la misma propiedad) por $\mathrm{m}^{2}$ de departamento, se calcula el costo total del proyecto al multiplicar dicho valor por el área de construcción. Posteriormente, se calcula el precio base del predio, a partir de una estimación del costo por $\mathrm{m}^{2}$ de construcción en altura en función de los acabados, y se aplica el método residual para la obtención del valor final de la edificación. El precio por $\mathrm{m}^{2}$ de suelo es el resultado de restar el costo estimado del proyecto del valor de la construcción y dividir para la superficie del lote. 


\subsection{Autocorrelación espacial}

Con la finalidad de visualizar la distribución espacial de los precios en todo el límite urbano de la ciudad, se realiza una interpolación espacial con el método de Kriging (ESRI, 2020). El Kriging emplea una variable de interés, en este caso el precio del suelo, para hacer estimaciones del valor posible en ubicaciones no muestreadas.

También, se emplea las estadísticas del I de Moran local y $\mathrm{Gi}^{*}$ con el fin de identificar posibles variaciones espaciales en los precios de suelo dados por la oferta y el avalúo municipal. La estadística $\mathrm{Gi}^{*}$ guía a identificar dónde se agrupan espacialmente los elementos con valores altos o bajos (puntos calientes/hotspots). Para representar un punto caliente estadísticamente significativo, una entidad debe tener un valor alto y también estar cerca de otros elementos con valores altos. La suma local para una entidad y sus vecinos se compara proporcionalmente con la suma de todos los elementos; cuando la suma local es muy diferente de la esperada, y esa diferencia es demasiado grande como para ser el resultado de una opción aleatoria, se obtiene como consecuencia una puntuación z estadísticamente significativa (ESRI, 2020). La estadística $\mathrm{Gi}^{*}$ se evalúa a través de la siguiente expresión:

$$
G_{i}^{*}=\frac{\sum_{j=1}^{n} w_{i, j} x_{j}-\bar{X} \sum_{j=1}^{n} w_{i, j}}{S \sqrt{\frac{\left[n \sum_{j=1}^{n} w_{i, j}^{2}-\left(\sum_{j=1}^{n} w_{i, j}\right)^{2}\right]}{n-1}}}
$$

Donde $x_{j}$ es el atributo del elemento $j, w_{i, j}$ es el peso espacial entre el elemento $i$ y $j, n$ es el número total de elementos.

$$
\begin{gathered}
\bar{X}=\frac{\sum_{j=1}^{n} x_{j}}{n} \\
S=\sqrt{\frac{\sum_{j=1}^{n} x_{j}^{2}}{n}-(\bar{X})^{2}}
\end{gathered}
$$

La estadística de I de Moran local permite, dado un conjunto de elementos ponderados, identificar puntos calientes, puntos fríos y valores atípicos espaciales estadísticamente significativos. El valor de autocorrelación espacial a través del I de Moran se establece como (ESRI, 2020):

$$
I_{i}=\frac{x_{i}-\bar{X}}{S_{i}^{2}} \sum_{j=1, j \neq i}^{n} w_{i, j}\left(x_{j}-\bar{X}\right)
$$

Donde $x i$ es el atributo del elemento $i, \bar{X}$ es la media de atributo correspondiente, $w_{i, j}$ es el peso espacial entre el elemento $i$ y $j$.

$$
S_{i}^{2}=\frac{\sum_{j=1, j \neq i}^{n}\left(x_{j}-\bar{X}\right)^{2}}{n-1}
$$

Con $n$ igual al número total de elementos.

En base a este análisis se establece la puntuación de $z$ (número de desviaciones estándar que hay por encima o por debajo de la media de población), y el valor $p$ (valor de probabilidad), ambos datos se emplean para identificar la significancia estadística del análisis.

La puntuación zIi se determina como:

$$
Z_{I i}=\frac{I_{i}-E\left[I_{i}\right]}{\sqrt{V\left[I_{i}\right]}}
$$

Con

$$
\begin{gathered}
E\left[I_{i}\right]=-\frac{\sum_{j=1, j \neq i}^{n} w_{i, j}}{n-1} \\
V\left[I_{i}\right]=E\left[I_{i}^{2}\right]-E\left[I_{i}\right]^{2} \\
E\left[I^{2}\right]=A-B \\
A=\frac{\left(n-b_{2 i}\right) \sum_{j=1, j \neq i}^{n} w_{i, j}^{2}}{n-1} \\
B=\frac{\left(2 b_{2 i}-n\right) \sum_{k=1, k \neq i}^{n} \sum_{h=1, h \neq i}^{n} w_{i, k} w_{i, h}}{n-1} \\
b_{2 i}=\frac{\sum_{i=1, i \neq j}^{n}\left(x_{i}-\bar{X}\right)^{4}}{\left(\sum_{i=1, i \neq j}^{n}\left(x_{i}-\bar{X}\right)^{2}\right)^{2}}
\end{gathered}
$$

Un valor positivo para el I de Moran indica que una entidad tiene elementos vecinos con valores de atributo altos o bajos similares, representando un grupo. Un valor negativo para I representa que un elemento tiene elementos vecinos con valores diferentes; el cual puede ser un valor atípico. En ambos casos, el valor $p$ para el elemento debe ser lo suficientemente pequeño para que el grupo o el valor atípico se consideren estadísticamente significativos. El grupo o valor atípico distingue entre un grupo de valores altos $(\mathrm{HH})$, un grupo de valores bajos (LL), un valor atípico en el que un valor alto está rodeado por valores bajos (HL) y un valor atípico en el que un valor bajo está cercado por valores altos (LH). La importancia estadística se establece en un nivel de confianza del $95 \%$.

\section{RESULTADOS Y DISCUSIONES}

En base al análisis estadístico de muestreo por sector de planeamiento, considerando el tamaño de la población $(\mathrm{N}=100,203)$, nivel de significancia $(\mathrm{z}=1.96,95 \%$ confiabilidad), error estimado $(\mathrm{e}=4 \%)$, probabilidad de éxito $(\mathrm{p}=0.5)$ y fracaso $(\mathrm{q}=0.5)$, resulta el número de muestras $n=594$. Se debe considerar que, a diferencia que otros componentes de estudios, los lotes en venta se basan en el deseo de vender de sus propietarios, lo cual puede limitar el número de muestras disponibles en cada sector. Sin embargo, en el periodo de levantamiento, la oferta inmobiliaria fue mayor y se ajustó a lo establecido en la muestra, recolectándose 1493 precios del mercado. En aquellas zonas donde no se alcanzó el número de datos establecidos, fundamentalmente, se expresan usos forestales por inundaciones y limitaciones topográficas, lo que disminuye la oferta inmobiliaria. De este total, los lotes que se pueden correlacionar con información oficial del municipio fueron 1058, esto debido a que se cuenta con la clave catastral para estos lotes que corresponden a la registrada en el municipio. De este total se verificó que las áreas registradas en el municipio y las informadas por los oferentes sean similares, con una variación máxima del 15\%, quedando 994 predios. En la Figura 1 se presenta 


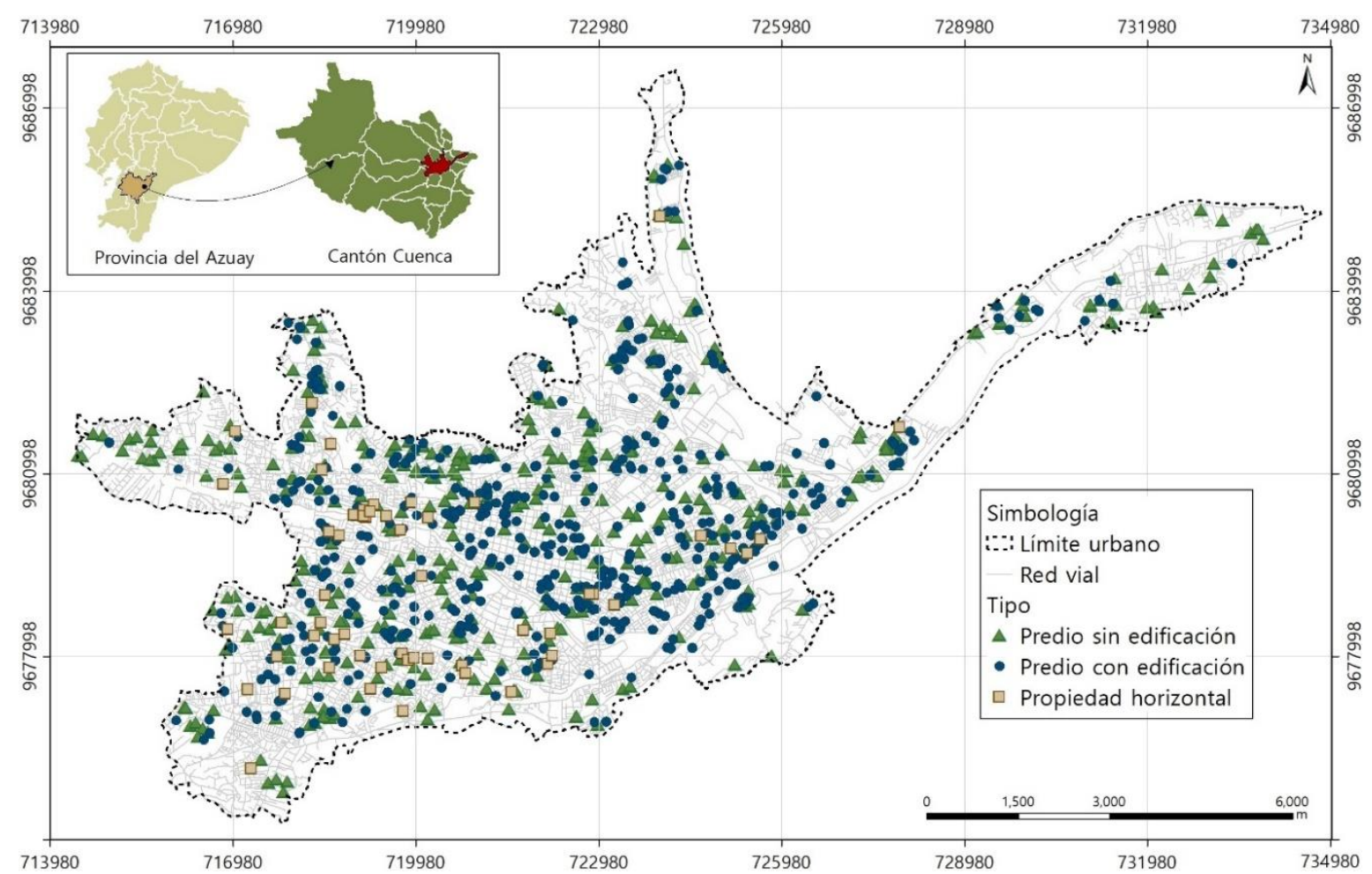

Figura 1. Distribución espacial de los 994 datos de precios del suelo en el área urbana de Cuenca.

la ubicación espacial de los lotes seleccionados en función de las características de ocupación del lote. Se tiene información de precios en toda el área urbana de la ciudad, excluyendo los sectores especiales. El número de predios sin edificación es de 421, con edificación 518 y propiedad horizontal 55. Se puede indicar que los predios de edificación horizontal se encuentran ubicados en ciertos sectores, excluyendo, por ejemplo, el centro y Este del área de estudio.

Tabla 1. Valores estadísticos del precio del suelo según el avalúo municipal y precios de oferta del mercado, para 994 predios en la ciudad de Cuenca.

\begin{tabular}{lrr}
\hline $\begin{array}{l}\text { Estadísticos } \\
\text { (USD/m2) }\end{array}$ & $\begin{array}{c}\text { Avalúo } \\
\text { municipal }\end{array}$ & $\begin{array}{c}\text { Precios del } \\
\text { mercado }\end{array}$ \\
\hline Media & 244.24 & 451.04 \\
Error típico & 4.67 & 7.29 \\
Mediana & 221.85 & 405.24 \\
Desviación estándar & 147.29 & 229.95 \\
Mínimo & 12.84 & 31.19 \\
Máximo & 734.36 & $1,839.95$ \\
\hline
\end{tabular}

Los resultados estadísticos del avalúo municipal y los precios de oferta del suelo se presentan en la Tabla 1. Los precios del suelo según el avalúo municipal tienen una media de $244.24 \mathrm{USD} / \mathrm{m}^{2}$, mientras que los precios de mercado tienen un valor de $451.04 \mathrm{USD} / \mathrm{m}^{2}$, lo que representa una relación de $54.15 \%$ del avalúo con respecto al precio del mercado. Este porcentaje está en proporción con el $50 \%$ determinado en un análisis realizado a 100 predios en la ciudad de Cuenca (Quille \& Quizhpi, 2011). Los valores municipales a menudo se establecen por debajo de los valores de mercado. En Alemania, los valores municipales se establecen en el $33 \%$ de los valores de mercado, mientras que en otros países el porcentaje es mayor. En Corea, una reforma fiscal a la propiedad consideró alinear los valores imponibles con los valores de mercado, pero los obstáculos políticos mantienen el porcentaje entre el 60 y $70 \%$ (Blöchliger, 2015).

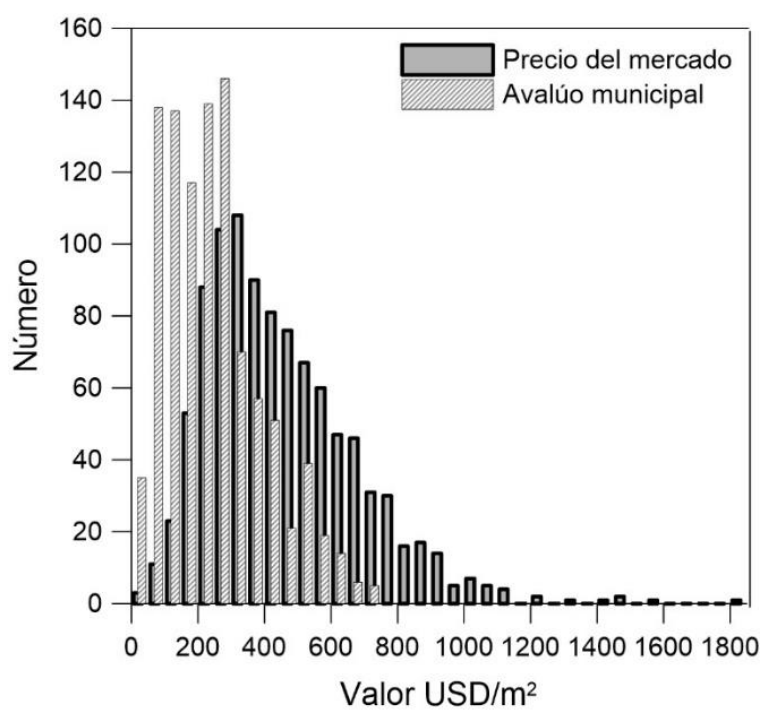

Figura 2. Histogramas de precios del suelo según el avalúo municipal y la oferta de mercado

En la Figura 2 se expone los histogramas de los valores correspondientes al avalúo municipal y precios de la oferta de mercado. Se observa que el histograma del avalúo municipal es más estrecho, con la mayoría de valores centrados en los 50 y $350 \mathrm{USD} / \mathrm{m}^{2}$, mientras que para los 


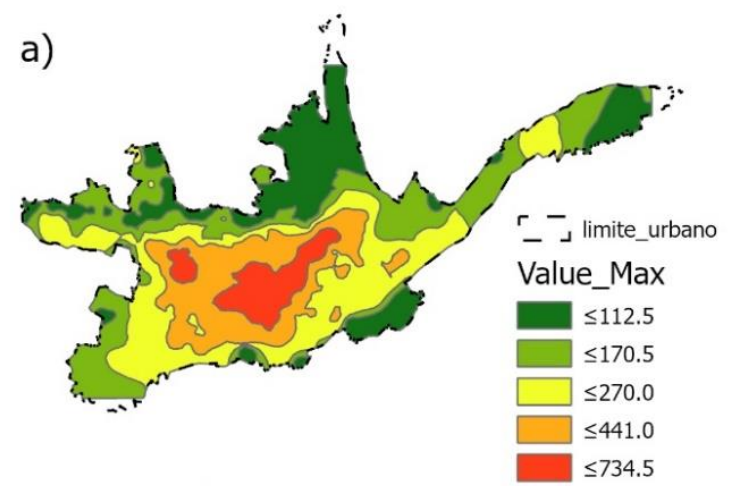

Avalúo Municipal

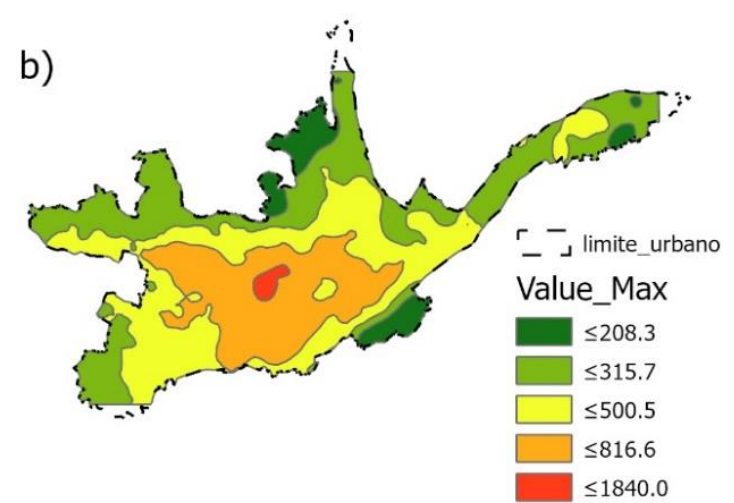

Precio del Mercado

Figura 3. Mapas interpolados mediante Kriging, a) avalúos municipales, y b) precios de mercado.

Tabla 2. Valores estadísticos de los diferentes grupos de datos relacionando al precio del suelo según el avalúo municipal y la oferta del mercado.

\begin{tabular}{|c|c|c|c|c|c|c|}
\hline \multirow{2}{*}{$\begin{array}{l}\text { Estadísticos } \\
\left(\mathrm{USD} / \mathrm{m}^{2}\right)\end{array}$} & \multicolumn{2}{|c|}{ Sin edificación } & \multicolumn{2}{|c|}{ Con edificación } & \multicolumn{2}{|c|}{ Propiedad horizontal } \\
\hline & $\begin{array}{c}\text { Avalúo } \\
\text { municipal }\end{array}$ & $\begin{array}{c}\text { Oferta del } \\
\text { mercado }\end{array}$ & $\begin{array}{c}\text { Avalúo } \\
\text { municipal }\end{array}$ & $\begin{array}{l}\text { Oferta del } \\
\text { mercado }\end{array}$ & $\begin{array}{c}\text { Avalúo } \\
\text { municipal }\end{array}$ & $\begin{array}{c}\text { Oferta del } \\
\text { mercado }\end{array}$ \\
\hline Media & 212.77 & 405.97 & 264.14 & 474.22 & 297.72 & 577.62 \\
\hline Error típico & 7.00 & 10.29 & 6.40 & 10.44 & 19.54 & 29.43 \\
\hline Mediana & 180.41 & 358.92 & 249.39 & 430.91 & 286.29 & 585.24 \\
\hline Desviación estándar & 143.70 & 211.11 & 145.57 & 237.66 & 144.95 & 218.25 \\
\hline Mínimo & 12.84 & 31.19 & 19.01 & 65.80 & 49.90 & 194.57 \\
\hline Máximo & 653.66 & $1,319.63$ & 734.36 & $1,839.95$ & 705.70 & $1,129.48$ \\
\hline Número de datos & \multicolumn{2}{|c|}{421} & \multicolumn{2}{|c|}{518} & \multicolumn{2}{|c|}{55} \\
\hline
\end{tabular}

Tabla 3. Valores estadísticos para la relación entre el precio del suelo según la oferta del mercado $(\mathrm{O})$ y el avalúo municipal (A).

\begin{tabular}{lcccc}
\hline $\begin{array}{l}\text { Estadísticos } \\
\text { Relación O/A }\end{array}$ & Sin edificación & $\begin{array}{c}\text { Con } \\
\text { edificación }\end{array}$ & $\begin{array}{c}\text { Propiedad } \\
\text { horizontal }\end{array}$ & Total \\
\hline Media & 2.42 & 2.16 & 2.21 & 2.27 \\
Error típico & 0.07 & 0.05 & 0.12 & 0.04 \\
Mediana & 2.01 & 1.81 & 2.09 & 1.93 \\
Desviación estándar & 1.46 & 1.24 & 0.92 & 1.33 \\
Mínimo & 0.59 & 0.38 & 0.61 & 0.38 \\
Máximo & 11.39 & 10.48 & 5.91 & 11.39 \\
Cuenta & 421 & 518 & 55 & 994 \\
\hline
\end{tabular}

precios de mercado la distribución es más extensa, indicando valores más dispersos. Se realizó una interpolación espacial de los valores, empleando el método Kriging, tanto para datos de avalúo como para precios de mercado, con el fin de obtener un mapa general del área de estudio que evidencia el comportamiento del mercado de suelo.

La Figura 3 se presenta las superficies interpoladas, tanto para los datos del precio del suelo del avalúo municipal como para precios del mercado levantados en este estudio. Se observa que el avalúo (Fig. 3a) tiene mayores picos en ciertas zonas, indicado que existe diferencias mayores; en el centro de la ciudad donde se presentan los valores más altos, la superficie estimada tiene una concentración más definida. Hacia el límite urbano, los precios del suelo van disminuyendo, sin embargo, no se visibiliza un patrón específico. Los precios de menor valor, en el caso del avalúo, se distribuyen espacialmente de manera similar, aunque en un área más extensa y en ciertas zonas puntuales, en comparación con los menores valores registrados de la oferta del mercado. La forma que se representa el mapa de avalúos responde a la discretización realizada en función de la sectorización en zonas homogéneas, esto se relaciona con el plano de valor del suelo del bienio 2020-2021. Para determinar las zonas homogéneas en los catastros, generalmente, se considera la dotación de servicios públicos, el uso del suelo, aspectos socio económicos, entre otros. En el caso de los precios de oferta (Fig. 3b) se evidencia una distribución de precios más uniforme en toda la zona urbana, pero de igual manera con la mayoría de precios más altos en el centro del área urbana. Estas diferencias se pueden deber a las dificultades que tienen los municipios de mantener un catastro vigente con información cartográfica y temática actualizada, lo que demanda altos costos, por lo que se requiere trabajar en nuevas metodologías que agiliten el proceso para la valuación masiva del suelo urbano. 
Para analizar posibles variaciones de los precios en base al tipo de predio levantado, en la Tabla 2, se presenta, por separado, los estadísticos de avalúos y ofertas en función del tipo del predio, es decir, entre los predios sin edificación, con edificación y propiedad horizontal. La diferencia en las medias se puede validar estadísticamente mediante la prueba Student t-test. Para los precios de mercado, de la prueba para dos muestras suponiendo varianzas iguales, entre predios sin y con edificación, el valor de probabilidad es $4.8 \mathrm{E}-06<0.05$, lo que indica que la hipótesis nula es rechazada, mostrando una diferencia significativa al 5\% entre las medias. Para el caso de predios sin edificación y propiedad horizontal, la prueba muestra, de igual manera, una diferencia de medias con probabilidad de 2.8E-08, mucho menor al valor crítico. Para el caso de predios con edificación y propiedad horizontal, la prueba rechaza, así también, la hipótesis de medias iguales con un valor de $2.1 \mathrm{E}-3<0.05$. Este análisis indica que los grupos de valores para las tres categorías son diferentes en el caso de los precios de mercado.

Para los precios del avalúo municipal, el valor de probabilidad del Student t-test es de $8.0 \mathrm{E}-08<0.05$, lo cual indica que la hipótesis nula es rechazada para los predios $\sin$ y con edificación. Para el caso de predios sin edificación y propiedad horizontal, la prueba indica, de igual manera, una diferencia de medias con probabilidad de 4.5E-05. Para el caso de predios con edificación y propiedad horizontal, la hipótesis nula, en este caso, no se rechaza con un valor de $0.1>0.05$, lo que muestra que las medias de estos dos grupos son similares según sus grupos de análisis. Los predios del avalúo sin edificación tienen una media de $212.77 \mathrm{USD} / \mathrm{m}^{2}$, con edificación 264.14 y propiedad horizontal 297.72 , lo que representa un incremento con respecto al primero de $24.14 \%$ y $39.93 \%$, respectivamente. En el caso de los precios de mercado, la media para predios sin edificación es de $405.97 \mathrm{USD} / \mathrm{m}^{2}$, con edificación 474.22 y propiedad horizontal 577.62, representado un $16.81 \%$ y $42.28 \%$, respectivamente, con respecto a los predios sin edificación.

La relación del precio de suelo dado por el avalúo y el precio de mercado se presenta en la Tabla 3. En este caso, no se determina una diferencia en las medias, según los análisis de la prueba Student t-test, por lo que el análisis de resultados se realiza de forma conjunta. La media total de la relación O/A es de 2.27, indicando que el precio de mercado es más de dos veces aquel registrado en el avalúo municipal. Es de esperarse que el avalúo municipal sea menor que el comercial, sin embargo, se considera conveniente analizar el rango de valores de tales diferencias que pueden contribuir para mejorar la recaudación de impuestos (beneficioso para el gobierno local) y en caso de expropiaciones (beneficio para el propietario), entre otras transacciones que se basan en el avalúo. Es interesante observar que, la diferencia puede alcanzar a valores de 11.39 veces, lo que indica que se está beneficiando de alguna manera a los propietarios de esos lotes con los avalúos actuales o existe una fuerte especulación del suelo urbano. O en su defecto, los valores de los bienes inmuebles no se han actualizado durante años o incluso décadas, como es el caso en varios países miembros de la Organización para la Cooperación y el Desarrollo Económico, lo que puede crear distorsiones e injusticia para los propietarios (Blöchliger, 2015). Así también, existen predios en los cuales el precio de venta es menor que el avalúo municipal, lo que puede deberse a factores propios del terreno, como características geográficas y físicas de los terrenos.

La Figura 4 presenta la distribución espacial de la relación entre el precio del suelo según la oferta del mercado con respecto al avalúo municipal (relación $\mathrm{O} / \mathrm{A}$ ). $\mathrm{Se}$ evidencian zonas en la que existen concentraciones de mayores diferencias de la relación O/A, esto se observa en

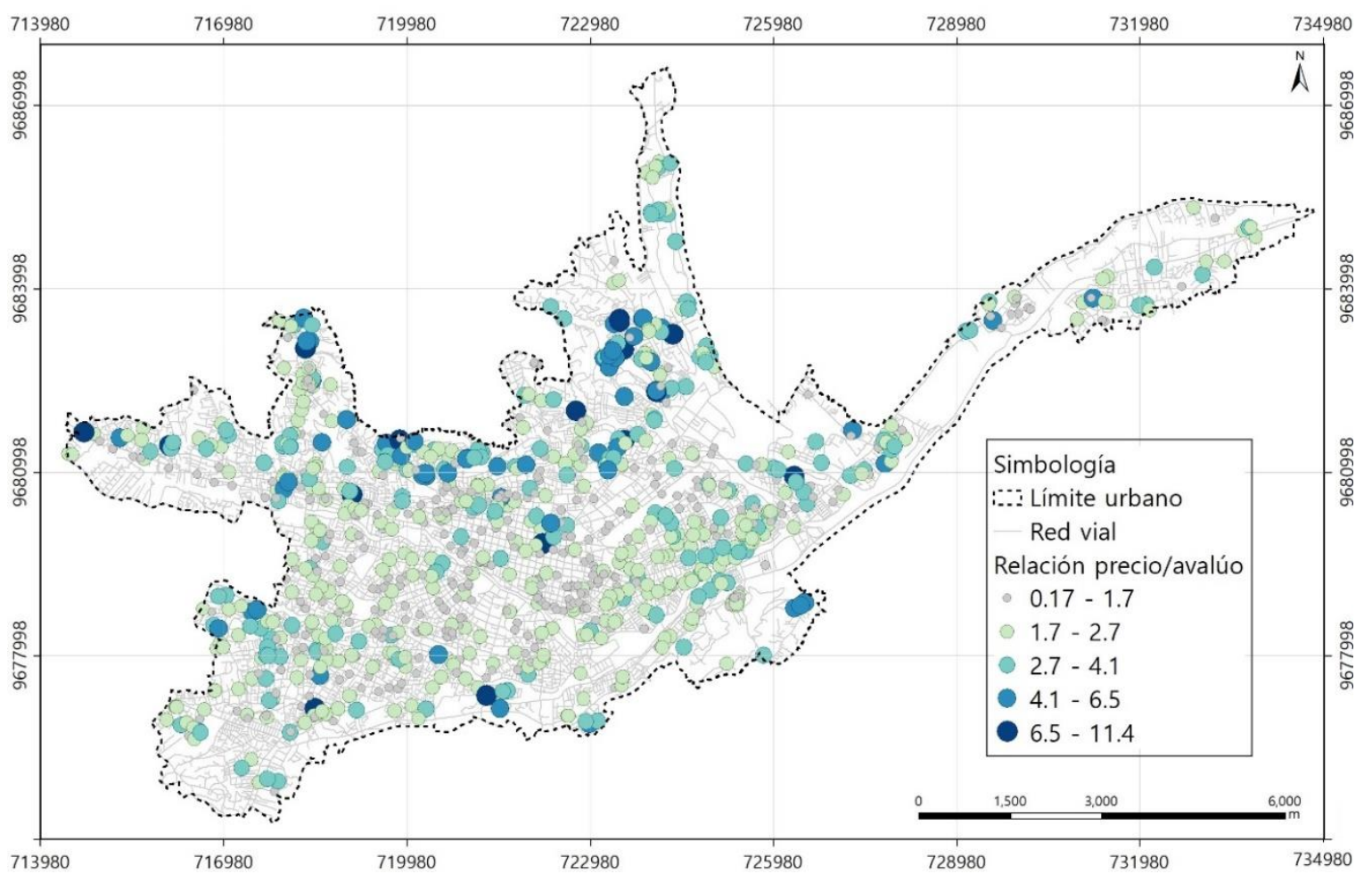

Figura 4. Distribución espacial de la relación entre el precio del suelo según la oferta del mercado y el avalúo municipal. 
la parte norte del área urbana. En toda la zona central se tiene varios predios con las menores diferencias en la relación O/A. Lo que podría evidenciar que el modelo empleado para el cálculo del avalúo catastral presenta un menor nivel de precisión en ciertas zonas. La variabilidad en relación con los precios de venta de los inmuebles puede significar la necesidad de ajustes globales o parciales en los modelos propuestos de valuación (Erba, 2007).

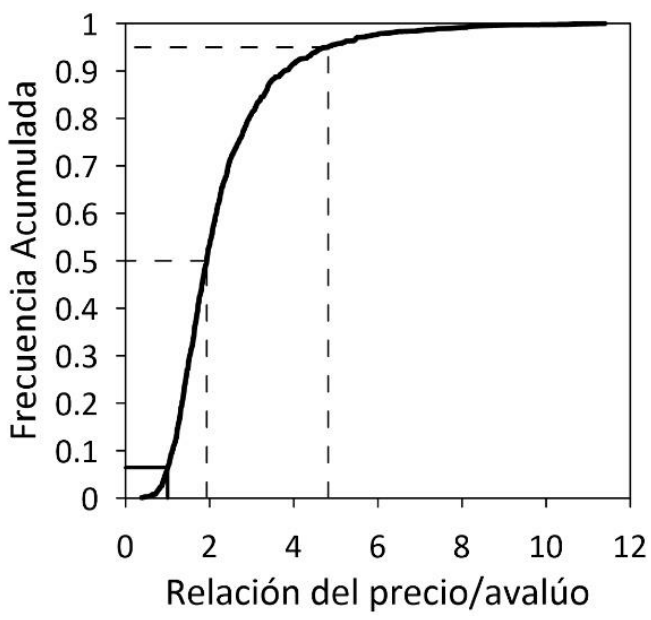

Figura 5. Frecuencia acumulada de la relación entre el precio del suelo dado por la oferta de mercado y el avalúo municipal.

En la Figura 5 se presenta la frecuencia acumulada de la relación precio de mercado versus avalúo. Se puede indicar que, el $6.4 \%$ de los datos analizados, es decir, 64 lotes de 994 tienen precios de oferta inferiores al avalúo municipal, con valores de la relación $\mathrm{O} / \mathrm{A}<1$. El percentil $50 \%$ con un valor de 1.93 , indica que 497 lotes tienen valores menores o iguales a 1.93 , dando 433 predios entre

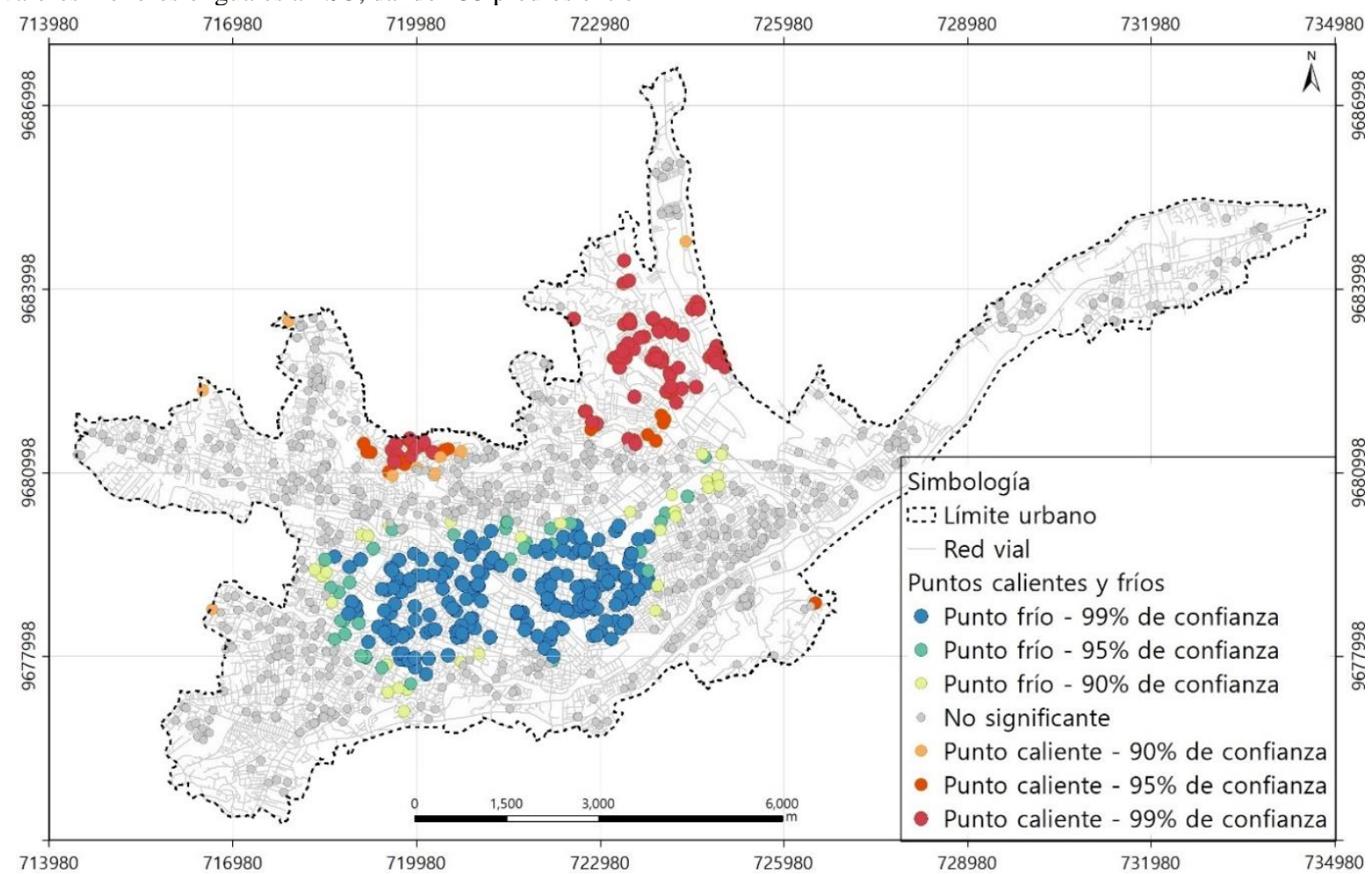

1 y 1.93 del avalúo (43.6\%). En el caso del percentil 95\%, 944 predios tienen valores menores o iguales a 4.82 y solamente el $5 \%$ (50 predios) tienen valores superiores al 4.82, con un máximo de 11.39 .

Con la finalidad de determinar diferencias acentuadas entre las relaciones de precios de mercado y el avalúo municipal, se determinaron puntos calientes según la metodología de $\mathrm{Gi}^{*}$. Si bien, los elementos con un valor alto o bajo son puntos de interés, sin embargo, es posible que no sean puntos calientes estadísticamente significativos, debido a que, para ser un punto caliente estadísticamente significativo, un elemento debe tener un valor alto o bajo y también estar rodeado por otros elementos con valores altos. En la Figura 6 se presenta el resultado obtenido para la estimación de puntos calientes bajo los criterios mencionados, considerando diferentes niveles de confiabilidad de 90, 95 y $99 \%$.

Se observa una concentración de puntos calientes (valores altos) en el norte del área y fríos (valores bajos) en el centro del área urbana. Los puntos fríos ubicados en el centro del mapa representan valores que tienen una diferencia menor en la relación precio de mercado y avalúo municipal que el resto de los datos de análisis, indicando que los precios de mercado son cercanos a los del avalúo, presentándose relaciones bajas. Los puntos calientes ubicados, principalmente, en el norte del mapa, indican que los precios de mercado son superiores en comparación con los otros datos de la zona urbana, por lo que los avalúos en estas zonas son muy inferiores a los requerimientos económicos de los propietarios. Lo que puede evidenciar un grado de especulación ya sea por las expectativas de desarrollo generadas en esos lugares, desconocimiento del vendedor del precio del lote, o incluso el involucramiento de intermediarios (Nuñez \& Schovelin, 2002). En la parte central se tienen las relaciones más bajas con un mínimo de 0.38 y un máximo de 3.37 .

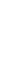

Figura 6. Puntos calientes y fríos en base a estadística Gi*. 


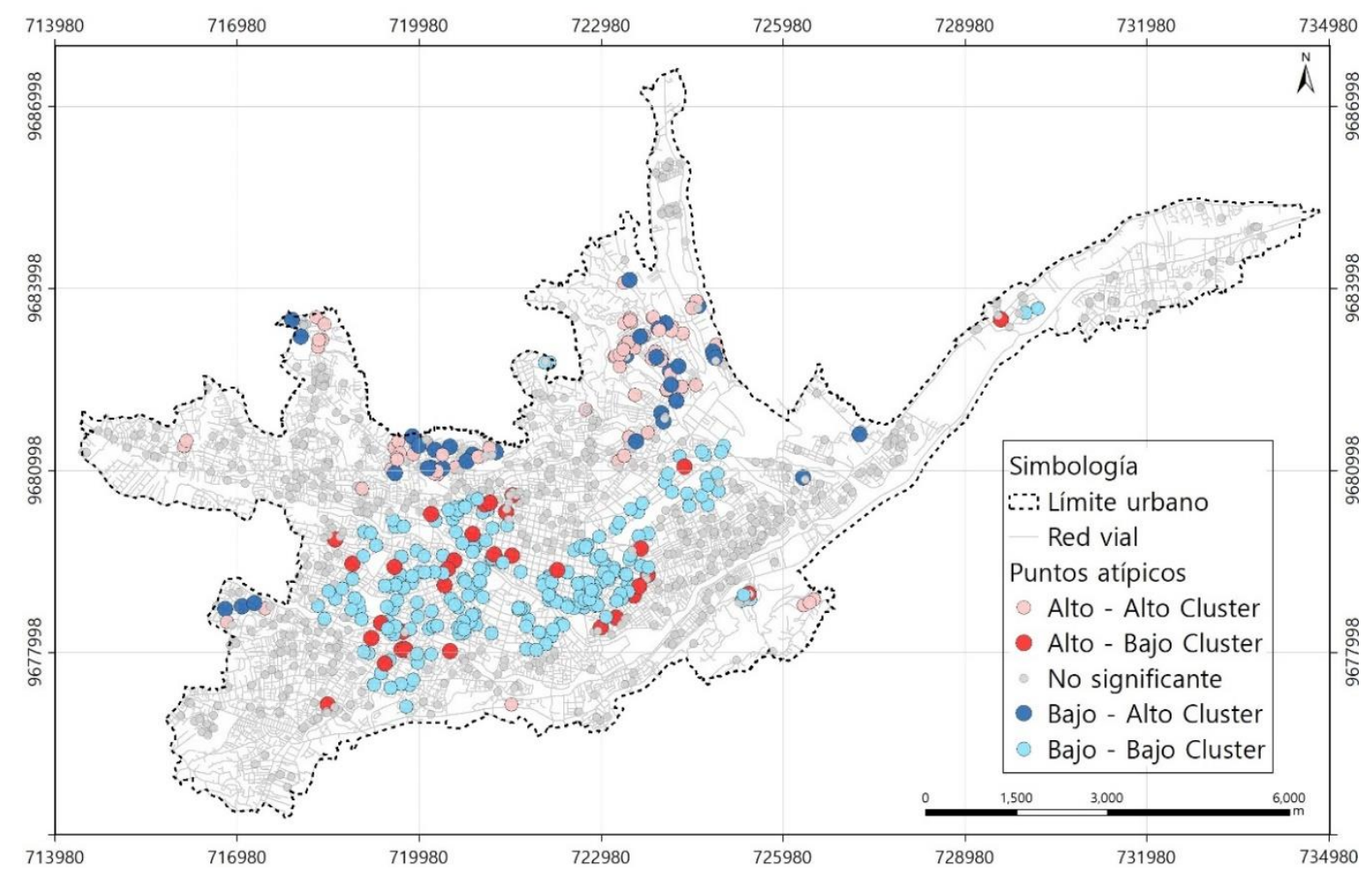

Figura 7. Puntos de interés en función de análisis de valores atípicos según I de Moran.

En base de un análisis de valores mediante el I de Moran, se identifican puntos que salen de las expectativas locales de las relaciones espaciales esperadas. La Figura 7 se presenta el mapa con la identificación de puntos atípicos dentro de cada zona de agrupación. Del análisis de autocorrelación espacial se han identificado valores atípicos dentro de los grupos. Se tiene 78 puntos $\mathrm{HH}$ (altoalto), indicando que corresponden a valores altos pero que están rodeando de valores también altos a su alrededor, estos se presentan en su mayoría en la zona norte del mapa. Dentro de la zona de relaciones altas, se presentan 34 puntos LH (bajo-alto) que muestran que son valores menores a los esperados en la zona de análisis. En el centro del mapa se presenta la mayoría de los valores de relación bajo-bajo LL (218) y algunos puntos (32) salen de este comportamiento de valores bajos, identificados como HL (alto-bajo), mostrando valores más altos de los esperados en estas zonas. En general, lo que permite identificar este análisis es la existencia de valores que salen del comportamiento esperado en cada sector, lo que debería generar estudios posteriores con la finalidad de entender de mejor manera este comportamiento. La implementación del impuesto a la propiedad sigue siendo difícil para los países en desarrollo donde los desafíos políticos son las deficiencias administrativas, financieras y técnicas de la autoridad tributaria; falta de datos legítimos, un sistema de valoración adecuado y voluntad política; mercados inmobiliarios inmaduros; y sistemas de registro, adquisiciones, legislación e hipotecas subdesarrollados (Hoque, 2019).

\section{CONCLUSIONES}

La recaudación de impuestos por concepto del suelo se basa en planos de valor del suelo urbano, los mismos que, en la mayoría de casos, son elaborados en base de la información de los precios dados por la oferta del mercado. Si bien, los municipios reducen, en cierta medida, los valores de mercado, no se han identificado procedimientos claramente definidos para tal fin. La metodología propuesta por el COOTAD para la definición del precio del suelo en base de comparación con precios de venta de inmuebles de condiciones similares $u$ homogéneas del mismo sector, abre la puerta a que el mercado rija en un bien tan importante para el desarrollo de los ciudadanos, como es el suelo. Por otro lado, al no existir un control en el precio que pueda solicitar un propietario por su lote, esto contribuye a la especulación del precio, basado en la oferta y la demanda del mercado. En base a una comparación de 994 precios del mercado de lotes en venta en el área urbana de Cuenca, se ha determinado la relación del precio del suelo dado por la oferta del mercado con respecto al avalúo municipal del terreno. Se determinó que, en promedio, el precio del suelo dado por el avalúo municipal es el $54.15 \%$ con respecto al precio del mercado, observándose que, el avalúo catastral es subvalorado en ciertos sectores. Se identificó que, dependiendo del tipo de lote; sin edificación, con edificación o propiedad horizontal, los precios difieren. Los precios del suelo dados por la oferta de mercado para predios con edificación son un $24.14 \%$ mayores a aquellos sin edificación, para propiedad horizontal el aumento es de $39.93 \%$, lo que indica que los propietarios recargan un precio adicional al suelo por concepto de edificación. Este incremento, debido a la construcción, también se observa en el avalúo municipal con un $16.81 \%$ para predios con edificación y $42.28 \%$ para los de propiedad horizontal.

Se determinó que la gran mayoría de los lotes analizados (93.6\%) tienen precios de suelos superiores a los estimados por el avalúo municipal, existiendo apenas un $6.4 \%$ de precios de oferta inferiores al avalúo municipal, 
que puede estar subvalorando la edificación y gravando más al suelo. La mitad de los propietarios que están vendiendo sus terrenos han solicitado precios que van desde 1.93 a 11.39 veces el precio del avalúo municipal, lo que puede evidenciar, entre otros aspectos, la especulación del suelo. De aquí, se considera necesario revisar el avalúo municipal, empleando una metodología adecuada para tal fin. Las diferencias obtenidas entre los valores dados por la oferta de mercado y el avalúo catastral, demuestra la necesidad de revisar la metodología empleada con fines catastrales a fin de identificar un modelo que se ajuste de mejor forma a la realidad de la ciudad, por ejemplo, a través de modelos predictivos que permitan identificar las variables que determinan el precio del suelo en la ciudad de Cuenca.

En base a un análisis de autocorrelación espacial se observó que la distribución de los precios dados por la oferta del mercado es más homogénea que aquella dada por el municipio. Se han determinado zonas en donde las diferencias en el precio del suelo dado por la oferta y el avalúo son mayores, principalmente en zonas hacia el norte de la ciudad, indicando que el avalúo en dichos sectores debe ser revisado y por lo tanto actualizar el plano de valor del suelo, puesto que se puede estar beneficiando a un cierto sector de la población. Así también, se identificaron valores atípicos, lo cual indican que existen propietarios que solicitan precios fuera de lo esperado según el comportamiento de los predios vecinos, lo que se puede deber a pura especulación del precio.

Es importante mencionar que aspectos externos como: localización del sitio, servicios cercanos, infraestructura, áreas verdes, entre otros factores, que influyen en el precio esperado por el propietario, están fuera del análisis del presente estudio. Así también, los municipios contemplan diferentes factores de reducción o aumento del precio del valor referencial según condiciones físicas de los terrenos. Sin embargo, estos aspectos indicados afectan en igual magnitud a los precios dados por la oferta de mercado que los considerados en el avalúo, por lo que los análisis realizados en el presente estudio son válidos.

Debido a que en una misma zona se presentan diferencias de relaciones, esto permite concluir que existe un aspecto especulativo en la ganancia esperada por un lote. Este análisis permite también identificar que el actual plano de valor del suelo subestima los valores que en la realidad se solicitan por los predios, independiente de los factores de reducción que el municipio maneja.

Es necesario avanzar en el desarrollo de metodologías de valuación masivas de suelo que permitan a las municipalidades: reducir costos, mantener la credibilidad de los ciudadanos y garantizar la aceptación de los valores e impuestos. El presente estudio pretende llamar la atención a los municipios del país para que empleen herramientas más técnico - científicas para la determinación del valor del suelo, sin perjudicar la recaudación de impuestos por subvalorar los precios de los predios, ni perjudicar a los propietarios con una sobrevaloración de los mismos.

\section{AGRADECIMIENTO}

Los autores desean agradecer el apoyo emitido por la Dirección de Investigación de la Universidad de Cuenca a través del aval al proyecto "Aplicación de la teoría de la renta de la tierra en la valuación del suelo urbano con fines catastrales para la gestión de las municipalidades en el Ecuador". Así también, al Arq. Alfredo Ordoñez, docente de la Universidad de Cuenca, por su aporte referente al análisis de precios de construcción.

\section{REFERENCIAS}

Blöchliger, H. (2015). Reforming the tax on immovable property: Taking care of the unloved. $O E C D$

Economics Department Working Papers, No. 1205, $30 \mathrm{p}$.

Borrero, O. (2010). Formación de los precios del suelo urbano. Cambridge, MA: Lincoln Institute of Land Policy. Educación a Distancia Programa para América Latina y el Caribe. Capitulo 6. 40 págs. Obtenido de https://flacso.edu.ec/cite/media/2016/02/BorreroO_ND_Formacion-de-los-precios-del-suelo-urbano.pdf

Conway, D., Li, C. Q., Wolch, J., Kahle, C., Jerrett, M. (2010). A spatial autocorrelation approach for examining the effects of urban greenspace on residential property values. Journal of Real Estate Finance and Economics, 41, 150-169. https://doi.org/10.1007/s11146-008-9159-6

COOTAD. (2010). Código orgánico de organización territorial, autonomía y descentralización. QuitoEcuador: Presidencia de La Republica del Ecuador.

Erba, D. (2007). Catastro multifinalitario aplicado a la definición de políticas de suelo urbano. Cambridge, MA: Lincoln Institute of Land Policy. 448 p. Obtenido de https://epdf.pub/queue/catastro-multifinalitarioaplicado-a-la-definicion-de-politicas-de-suelourbano.html

Erba, D. (2013). Definición de políticas de suelo urbano en América Latina: teoría y práctica. Cambridge, MA: Lincoln Institute of Land Policy. 286 p. Obtenido de https://www.suelourbano.org/wp-content/uploads/ 2017/09/Lincoln-definicion-de-politicas-de-suelourbanas.pdf

ESRI. (2020, Marzo 10). An overview of the Spatial Statistics toolbox. Retrieved from https://desktop.arcgis.com/en/arcmap/10.3/tools/spatial -statistics-toolbox/an-overview-of-the-spatial-statisticstoolbox.htm

GAD Cuenca. (1998). Reforma, actualizacion, complementacion y codificacion de la ordenanza que sanciona el plan de ordenamiento territorial del Canton Cuenca. Cuenca, Ecuador: Concejo Cantonal de Cuenca.

GAD Cuenca. (2019). Ordenanza de aprobación del plano de valor del suelo urbano, urbano parroquial y rural, de los valores de las tipologías de edificaciones, los factores de corrección del valor de la tierra y edificaciones y las tarifas para el bienio 2020-2021. Cuenca, Ecuador: Concejo Cantonal de Cuenca.

Granda, A., Cuesta, R., \& Villagomez, M. (2017). Modelo de valoración masiva de suelo urbano caso de estudio: ciudad de Tena, Ecuador. Geografía y Sistemas de Información Geográfica (GeoSIG), 9(9), 152-170. 
Hoque, M. S. (2019). Addressing property taxation challenges for developing countries: an application of a spatially based, weighted parametric valuation model. Journal of Property Tax Assessment \& Administration, $16(2), 15-40$.

Jaramillo, S. (2009). Hacia una teoría de la renta del suelo urbano. Santafé de Bogotá, Colombia: Ediciones Uniandes-Instituto Geográfico Agustín Codazzi.

Jiao, L., \& Liu, Y. (2012). Analyzing the spatial autocorrelation of regional urban datum land price. Geo-spatial Information Science, 15(4), 263-269. https://doi.org/10.1080/10095020.2012.714103

Kim, B., \& Kim, T. (2016). A study on estimation of land value using spatial statistics: Focusing on real transaction land prices in Korea. Sustainability, 8(3), 203, 14 p. https://doi.org/10.3390/su8030203

Larsen, R. J., \& Marx, M. L. (2006). An introduction to mathematical statistics and its applications $\left(4^{\text {th }} \mathrm{ed}.\right)$. Merseburg, Germany: SILO.PUB.

Liu, Z., Li, M., Sun, Y., \& Ma , W. (2006). Study on spatial autocorrelation of urban land price distribution in Changzhou city of Jiangsu Province. Chinese Geographical Science, 16, 160-164. https://doi.org/10.1007/s11769-006-0011-8

Lozano-Gracia, N., \& Anselin, L. (2012). Is the price right?: Assessing estimates of cadastral values for Bogota, Colombia. Regional Science Policy \& Practice, 4(4), 495-508. https://doi.org/10.1111/j.17577802.2012.01062.x
Nuñez, F., \& Schovelin, R. (2002). Modelo de precio de suelo urbano en el gran Concepción. Revista Ingeniería Industrial, 1(1), 47-58.

Paterson, R., \& Boyle, K. (2002). Out of sight, out of mind? Using GIS to incorporate visibility in hedonic property value models. Land Economics, 78(3), 417 425. https://doi.org/10.2307/3146899

Quille, G., \& Quizhpi, P. (2011). El catastro municipal y su aplicación en el ámbito tributario. El valor de la propiedad. Tésis, 67 págs. Cuenca, Ecuador: Universidad Politécnica Salesiana.

Scheaffer, Mendenhall, Ott, \& Gerow. (2012). Elementary survey sampling ( $7^{\text {th }}$ ed.). 480 p. Boston, MA: Brooks/Cole, Cengage Learning.

Shapiro, E., Mackmin, D., \& Sams, G. (2013). Modern methods of valuation (11 $1^{\text {th }}$ ed.). 582 p. London, UK: Routledge and EG Books.

Womack, K. (2015). Real options and urban land values: A review of the literature. Journal of Real Estate Literature, 23(1), 53-63. https;//doi.org/10.1080/ 10835547.2015.12090398

Xiao, Y., Chen, X., Li, Q., Yu, X., Chen, J., \& Guo, J. (2017). Exploring determinants of housing prices in Beijing: An enhanced hedonic regression with open access POI data. International Journal of GeoInformation, 6(358), 1-12. https://doi.org/10.3390/ ijgi6110358 